\title{
Computational Analysis of Histological Images of Tissue Engineered Cartilage for Evaluation of Scaffold Cell Migration
}

\author{
${ }^{1}$ Thomas Lepiarz, ${ }^{1}$ Ulla Wenzel, ${ }^{1}$ Michael Munz, ${ }^{2}$ Katja Hasch, ${ }^{2}$ Eva Goldberg-Bockhorn, ${ }^{2,3}$ Nicole Rotter, \\ ${ }^{1}$ Martin Hessling \\ ${ }^{1}$ Institute of Medical Engineering and Mechatronics, UIm University of \\ Applied Sciences, Germany \\ ${ }^{2}$ Department of Otorhinolaryngology, \\ Ulm University Medical Center, Ulm, Germany \\ ${ }^{3}$ Department of Otorhinolaryngology, \\ Mannheim University Medical Center, Mannheim, Germany \\ lepiarz@mail.hs-ulm.de,wenzel@hs-ulm.de,michael.munz@hs-ulm.de, Katja.Hasch@uniklinik-ulm.de, \\ eva.goldbera@uniklinik-ulm.de, Nicole.Rotter@uniklinik-ulm.de, hessling@hs-ulm.de
}

\begin{abstract}
Human chondrocytes were seeded on porcine collagen scaffolds and cultivated for up to six weeks in a cartilage bioreactor. To evaluate the influence of cultivation parameters on the proliferation and migration of the cells into the scaffolds, microscopic images from histological and immunohistochemical stainings were taken and digitalized. For evaluation of these pictures, image processing algorithms have been developed that enable quantitative conclusions with regards to aggrecan and collagen type I concentrations as well as the number of cell nuclei within the scaffold and respective migration depths. Furthermore, the number of scaffold lacunae and their orientation relative to the scaffold's surface can be determined. A total of 85 images of different cultivations under various conditions were processed and the results evaluated by an expert. Additionally, the findings were related to results of available conventional biochemical laboratory results. The outcomes showed very few minor flaws but were valid in most cases. Some findings - as the distribution of the total cell number between cells on the surface and inside the scaffold - are superior to conventional laboratory methods that do not give this insight. A further advantage compared to the established common expert evaluation of these images, is that this approach is faster and less dependent on the judgement of the individual expert and offers quantitative results. The software development will be continued and applied for further optimizing of cartilage culture conditions.
\end{abstract}

Keywords: image analysis; histological images; cartilage; scaffolds; bioreactor

\section{Introduction}

Structural and functional facial cartilage defects are mainly caused by congenital lesions, traumata and cancer. Multiple and complex surgical interventions are necessary to repair those defects [1]. Tissue 
Engineering (TE) with autologous cells is an alternative therapeutical approach. Human chondrocytes are seeded on porous scaffolds and cultivated to form new vital cartilage. During ex vivo culture cells proliferate and migrate into the scaffold prior to implanting the construct into the patient. Unfortunately, cultivating chondrocytes on 3D scaffolds to form new vital cartilage is very challenging and success is not guaranteed due to the complex influencing factors, which are not fully understood yet. Next to biocompatibility, biodegradability, biochemical and mechanical properties the structure of the scaffold is a decisive factor. Open and porous scaffolds are essential for nutrition, proliferation and migration of cells as well as for the development of new cartilage tissue [2,3]. Decellularized porcine cartilage scaffolds seem to be a promising material to restore cartilage defects. The decellularization process eliminates all immunogenic components of the cartilage in particular cells and glycosaminoglycans (GAG) so that a pure chondroconductive collagen scaffold remains [4]. To assess the quality of cell growth and migration it is common to take microscopic images from ultrathin sections of the preparations after immunostaining of selected components. These images are usually interpreted by experts whose evaluation depends on their skills and training. Furthermore, even a highly competent professional has difficulties quantifying values like average migration depth, cell numbers and concentrations or average lacunae properties. This makes a precise and objective diagnosis based on histological images an ongoing challenge for scientists.

A computer-aided diagnosis with image processing algorithms and techniques could be an alternative approach for the evaluation of histochemical images $[5,6]$ but has not been applied to chondrocyte migration into scaffolds so far. Among the advantages are the reproducibility of the results, the possibility of determining quantitative values for the comparison of images and the speed of an automated computer-based image evaluation.

Unfortunately, there are no standard procedures or standard algorithms for performing meaningful analyses. A relevant review about computational histological analysis of hematoxylin-eosin (H\&E), fluorescence and multispectral images is presented by Fuchs and Buhmann in [7]. The authors discuss the preprocessing of images and segmentation of different tissue components to distinguish relevant from irrelevant information. Those regions of interest (ROI) are characterized by defined textures or distinct colors and shades. For additional image processing mathematical-morphological operations are utilized which were first mentioned by George Matheron in [8]. Morphological processing operations require the interaction of the given image pixel with an external set of pixels which form a structure element (SE). They are utilized to remove insignificant structures by retaining the characteristics of an image.

In this paper a novel approach of segmenting histological images is presented that should offer objective and quantitative data from histochemically prepared cartilage sample images for improving future cartilage cultivation parameters. Ultrathin sections from decellularized porcine septal cartilage scaffolds seeded with human nasal chondrocytes were analyzed. The results of 85 image analyses were controlled by an expert and compared to the results of standard laboratory methods.

\section{Methods}

\subsection{Material and Histological Image Acquisition}

3D scaffolds of decellularized porcine nasal septum cartilage were seeded with human nasal chondrocytes and then cultivated in a bioreactor (BR) with automated nutrient and gas supply for up to 6 weeks. 
Thomas Lepiarz, Ulla Wenzel, Michael Munz, Katja Hasch, Eva Goldberg-Bockhorn, Nicole Rotter, Martin Hessling; Computational Analysis of Histological Images of Tissue Engineered Cartilage for Evaluation of Scaffold Cell Migration. Journal of Biomedical Engineering and Medical Imaging, Volume 4, No 6, December (2017), pp 1-15

Samples were taken from culture after 14, 28 and 42 days [9,10]. Additionally, statical cultivations without medium flow were conducted for comparison to the BR. In total three cultivation attempts were evaluated. The immunohistochemical staining procedures for aggrecan and collagen type II are presented in [11], for collagen type I and histological alcian blue staining in [12]. All samples were analyzed with a Zeiss Observer.D1 Microscope and visual recordings were performed with the Zeiss AxioCam MRc Camera with 1380x1040 pixels. For this purpose, the Zeiss software package AxioVision was employed. The images were taken with a tenfold microscope magnification by a skilled medical technician. Typical sample images with many chondrocytes in a cell pellet on top of the scaffolds can be found in Figure 1. It is also perceptible that the more important composition within the scaffold may be very different from the situation in the cell pellet.

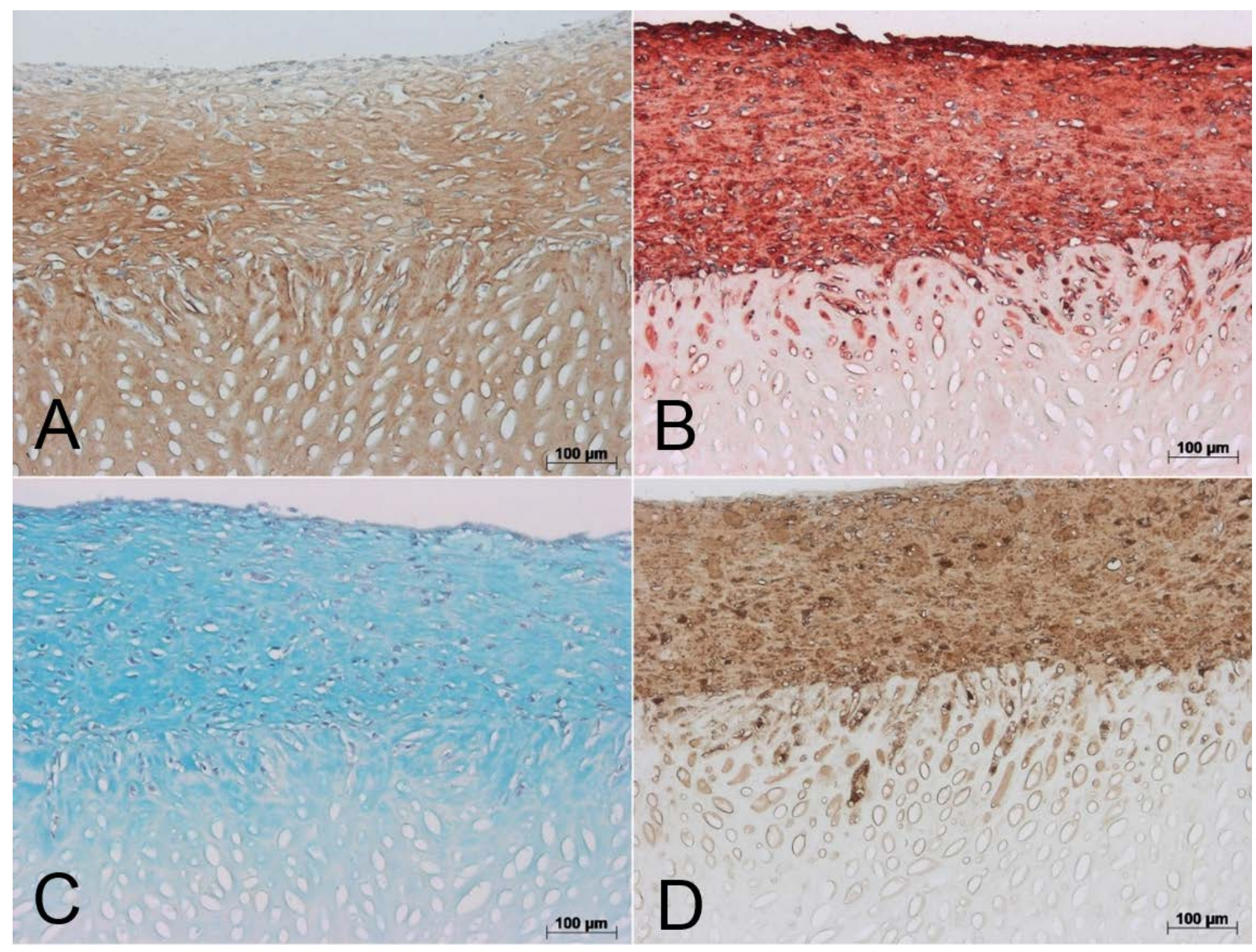

Figure 1: Examples of histological images of decellularized porcine cartilage scaffolds seeded with human chondrocytes. A: Immunohistochemical staining to detect collagen type II as component of the scaffold and synthesis product of the seeded cells (brown colouring). B: Tissue section after immunohistochemical staining of collagen type $\mathrm{I}$, which is produced by the seeded cells (red-brown colouring). C: Alcian blue staining reveals the typical blue coloring of the muco-polysaccharids produced by the chondrocytes and the darker cell nuclei whereas the scaffold's matrix is nearly transparent. D: Brown colouring indicates aggrecan production of the cells. 


\subsection{Image Processing}

The segmentation algorithm is divided into several stages and summarized in Figure 2. The algorithm was implemented and realized with the Image Processing Toolbox ${ }^{\mathrm{TM}}$ in MATLAB $^{\circledR}$, Version R2016b (The MathWorks, Inc.). For the observer's comprehension of every single process step a user interface was designed.

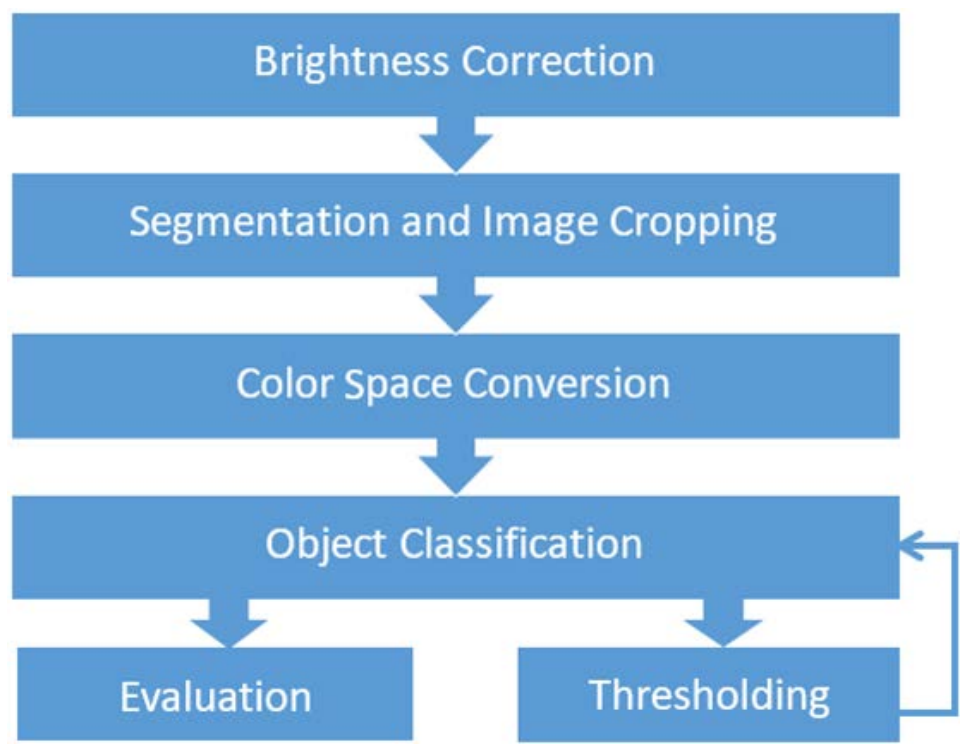

Figure 2: Summarized segmentation process.

\subsection{Brightness Correction}

Because of different illumination conditions during image acquisition and variations in the staining procedures the images differ in brightness and color intensities. To simplify the image analysis a first image processing step normalizes the images and reduces illumination and staining differences.

All pixels contain information about red, green and blue intensities. A region of interest (ROI) is manually selected, which complies with the background. This ROI with the size $w \times h$ is a subimage $\mathrm{J}$ of the original image $\mathrm{G}$.

The average red, green and blue channel values of the image $J$ are calculated:

$R_{\text {mean }}=\frac{1}{w h} \sum_{j=1}^{h} \sum_{j=1}^{w} J(i, j, 1), G_{\text {mean }}=\frac{1}{w h} \sum_{j=1}^{h} \sum_{j=1}^{w} J(i, j, 2), B_{\text {mean }}=\frac{1}{w h} \sum_{j=1}^{h} \sum_{j=1}^{w} J(i, j, 3)$

A new image $\mathrm{F}$ with size $w \times h$ is created containing the averaged values $R_{\text {mean }}, G_{\text {mean }}$ and $B_{\text {mean }}$ in any pixel. By subtracting $F$ from $G$, a new image $I$ is calculated as depicted in Figure 3. 
Thomas Lepiarz, Ulla Wenzel, Michael Munz, Katja Hasch, Eva Goldberg-Bockhorn, Nicole Rotter, Martin Hessling; Computational Analysis of Histological Images of Tissue Engineered Cartilage for Evaluation of Scaffold Cell Migration. Journal of Biomedical Engineering and Medical Imaging, Volume 4, No 6, December (2017), pp 1-15

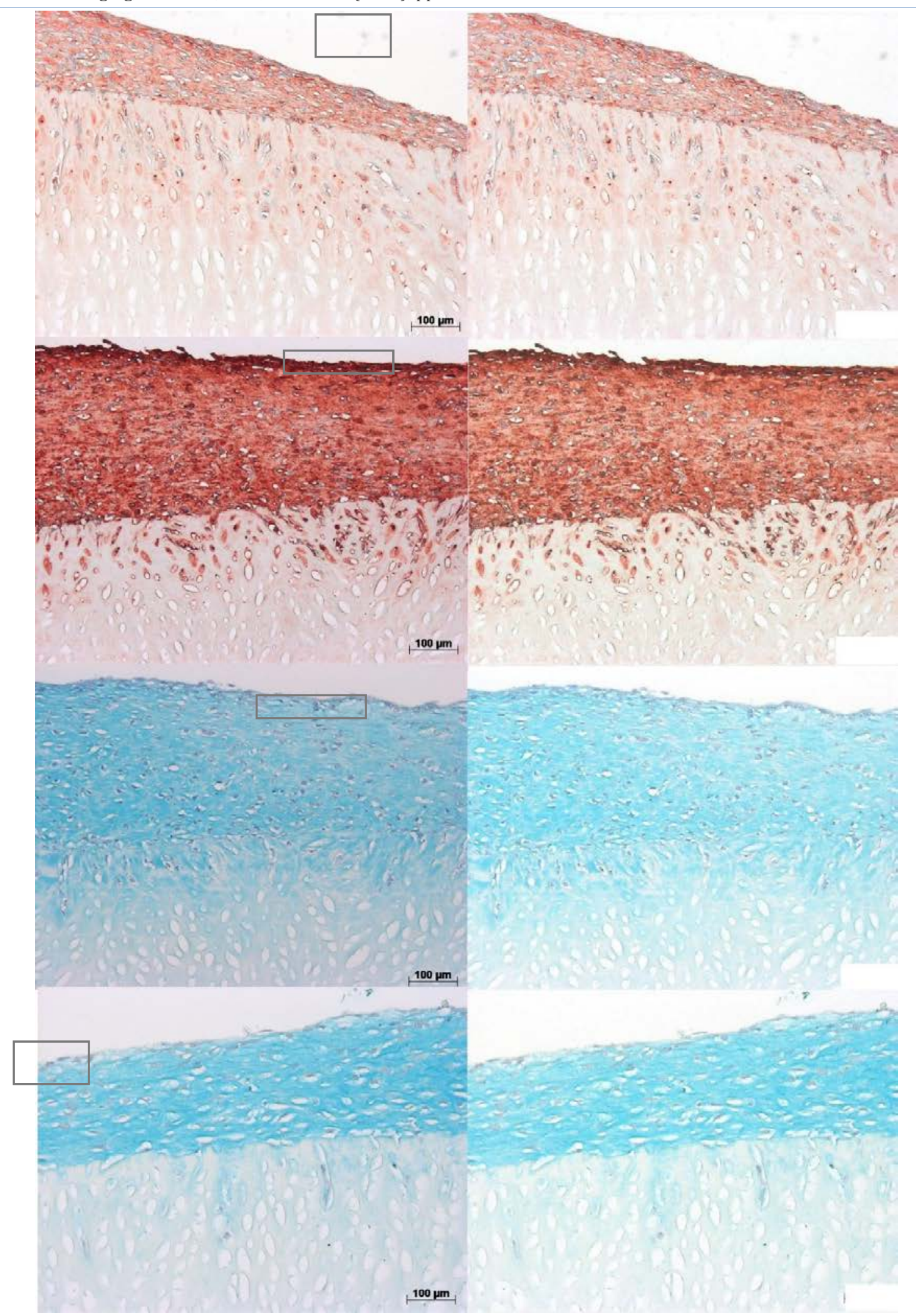

Figure 3: Four examples of brightness correction. In the two red images above collagen type I is stained. The images below depict alcian blue stained samples. For each kind of staining an example for a brighter and darker original image is given to prove that the normalized results are comparable. The green squares in the uncorrected pictures on the left side represent the area, which is averaged (right pictures). Scales in the lower right corner are removed because they interfere with the image analysis. 


\subsection{Segmentation and Image Cropping}

The aim of image segmentation is to highlight or differentiate objects from each other or from the environment. Here, it is necessary to separate the cell pellet on the surface of the scaffold from the scaffold matrix, in which the cells should migrate and differentiate. Therefore, several edge points of a $\mathrm{ROI}$ are manually selected in the image on the surface line of the scaffold. These ROI points are used as a polygon separation line. A binary mask is created with white pixels above and black pixels below this polygon. The binary mask is then employed for separating the scaffold (SF) from the cell pellet (CP) on top of the scaffold which results in two images I(SF) and I(CP), as illustrated in Figure 4.

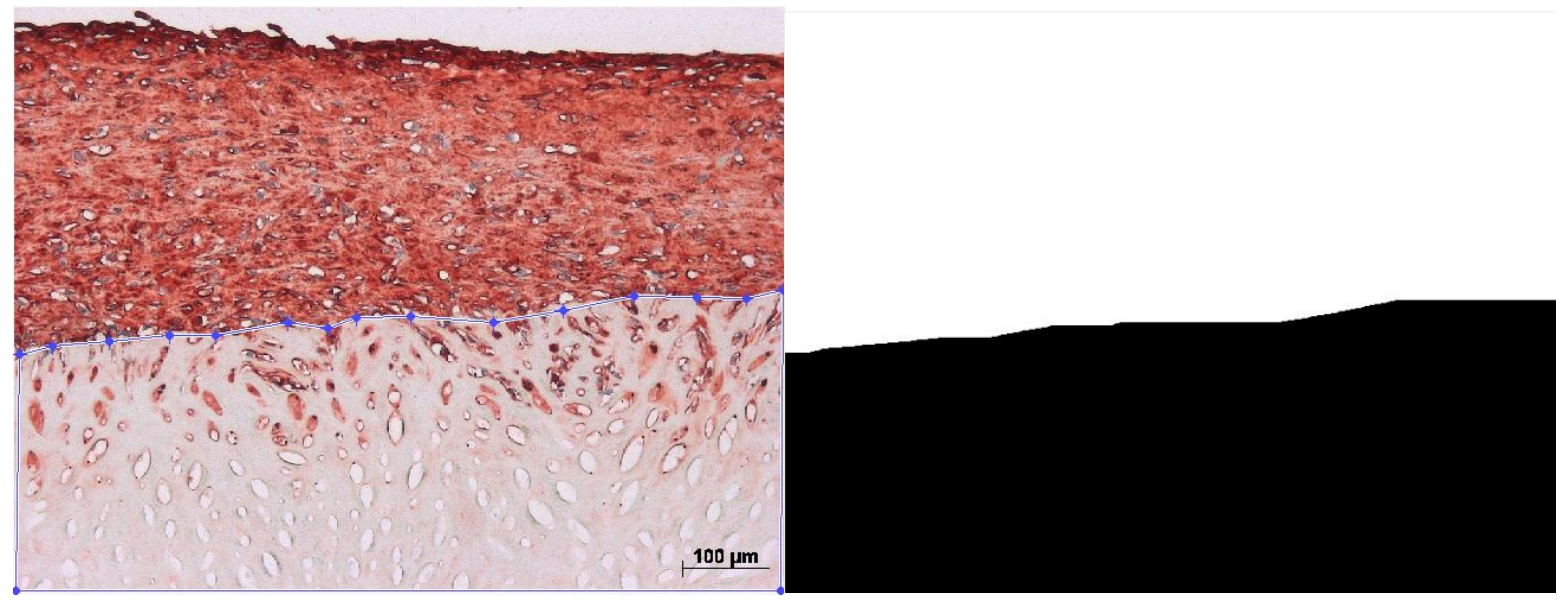

Figure 4: Blue dots and lines represent selected ROI (left) and the resulting binary image (right).

\subsection{Color Space Conversion}

For further segmentation, the images I(SF) and I(CP) are converted from RGB color space into the HSV color space. This transformation changes the pixel information from red, green and blue intensity into Hue $(H)$, Saturation $(S)$ and Value $(V)$, which represents the color, the purity of a color and the luminous intensity of a pixel. $\mathrm{H}$ is defined in a range between 0 and 360 whereas $\mathrm{S}$ and $\mathrm{V}$ are defined in a range between 0 and 1. The HSV color space is illustrated in Figure 5 and further explained in [13].

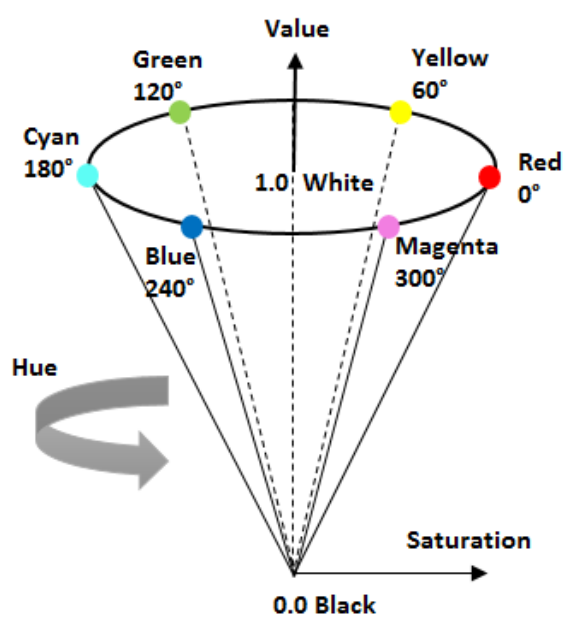

Figure 5: Illustration of HSV color space. 
Thomas Lepiarz, Ulla Wenzel, Michael Munz, Katja Hasch, Eva Goldberg-Bockhorn, Nicole Rotter, Martin Hessling; Computational Analysis of Histological Images of Tissue Engineered Cartilage for Evaluation of Scaffold Cell Migration. Journal of Biomedical Engineering and Medical Imaging, Volume 4, No 6, December (2017), pp 1-15

Active chondrocytes produce collagen type I \& II, glycosaminoglycans and aggrecan, with ratios depending on their differentiation status. The concentrations of these substances can be evaluated after the immunohistochemical staining and image analysis. Due to the unstained scaffold, which is bright and transparent, good contrast to the stained area is given. The specific stained area in the aggrecan and collagen type I stained images mark the ROIs, which hue information are narrowed in a defined range. For collagen type I the hue range is defined between 3 and 40 as well as between 350 and 358. For aggrecan and collagen type II the hue range is between 10 and 60 as well as 350 and 358. In Figure 6 two histograms are presented that show the hue distribution of two images. Because of the bright color of the staining methods, residues are left on the scaffold that are in the defined hue range of the ROI. The residues are excluded by defining the value range. For collagen type I and aggrecan, the value channel $V$ of every pixel with $V>0.8$ was set to zero. The value channel of every other pixel of the image that does not fit into the defined hue range is also set to zero. When converted back to the RGB image, all pixels with $V=0$ result in black pixels. The new RGB images are then converted into grayscale images that are binarized into I(SF) edited and I(CP) edited (Figure 7) with a small threshold value slightly above 0 . Every white pixel now represents the ROI. Since the area of a camera pixel corresponds to approximately $0.42 \mu \mathrm{m}^{2}$ in the sample, the size of the cell pellet and the area with produced aggrecan or collagen type I can be determined.

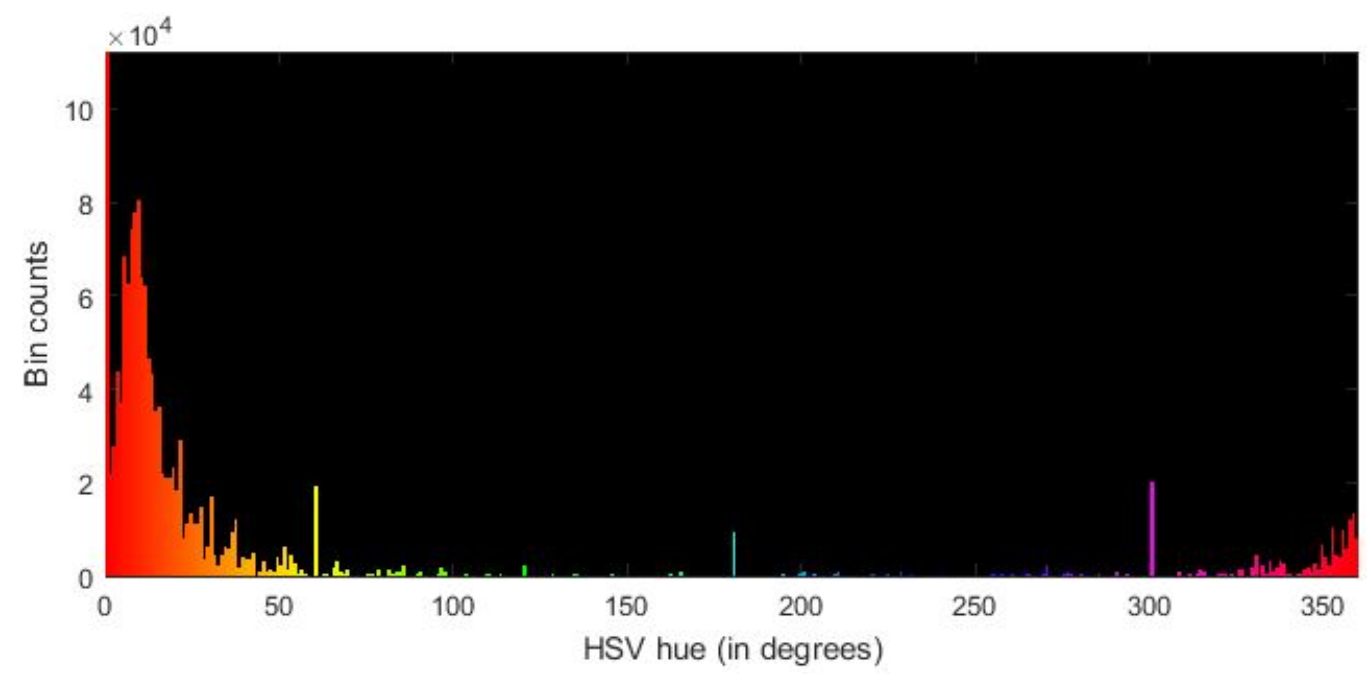




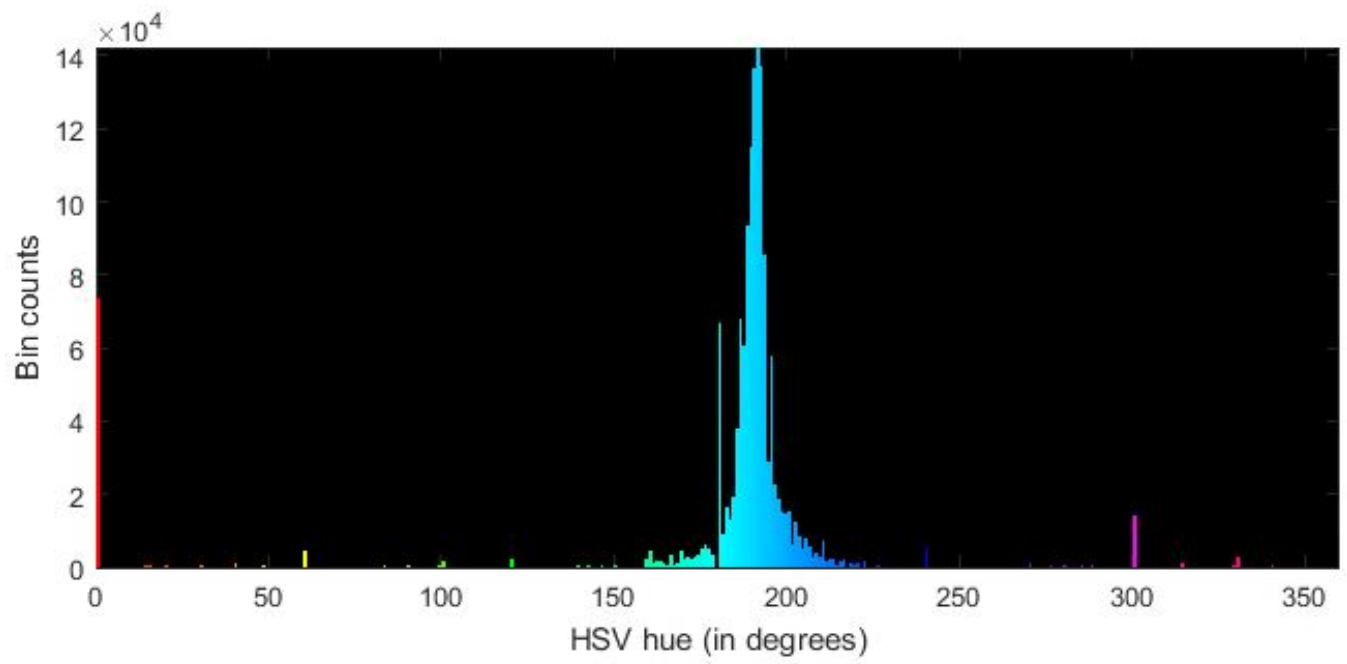

Figure 6: Hue histogram of a collagen type I image (top) and an alcian blue image (bottom).

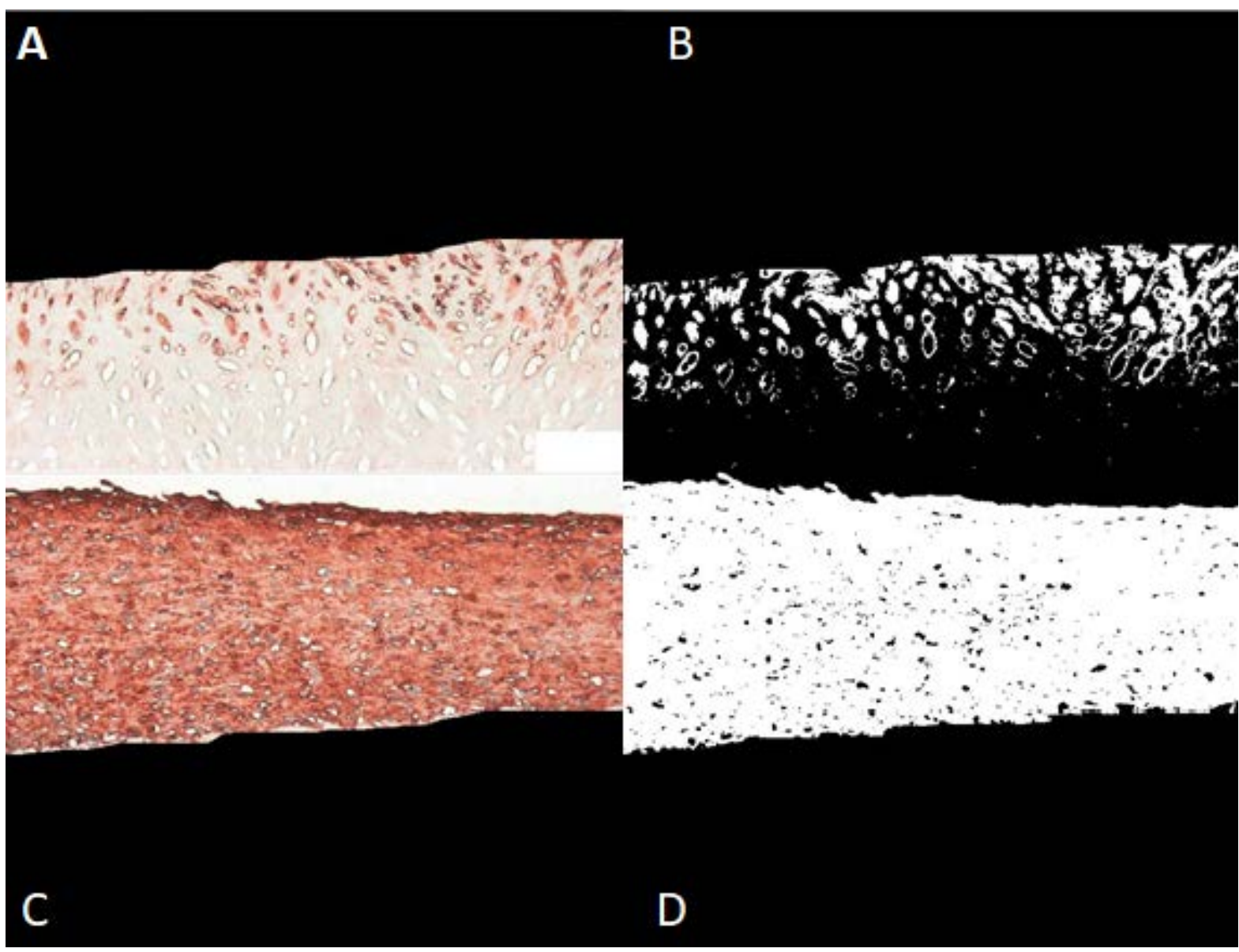

Figure 7: A: Image I(CP) shows the scaffold area with stained collagen type I, B: Image I(SF)edited depicts detected collagen type I in the scaffold as white pixels in the binary image, C: Image I(SF) represents cell pellet area with stained collagen type I, D: Image I(CP) edited illustrates detected collagen type I in the cell pellet as white pixels in the binary image. 
Thomas Lepiarz, Ulla Wenzel, Michael Munz, Katja Hasch, Eva Goldberg-Bockhorn, Nicole Rotter, Martin Hessling; Computational Analysis of Histological Images of Tissue Engineered Cartilage for Evaluation of Scaffold Cell Migration. Journal of Biomedical Engineering and Medical Imaging, Volume 4, No 6, December (2017), pp 1-15

\subsection{Cell Numbers and Migration Depths}

The number of cells inside the scaffold and their migration depths are very important values for the desired engineered cartilage. In order to assess the migration depth of the chondrocytes, cell nuclei have to be identified. Images of preparations stained with alcian blue turned out to be best for the detection of cell nuclei, which are blue stained in every image and overlaid by the other stains. To separate and detect the nuclei in alcian blue images, a range for value and saturation must be defined. The value of every other pixel, which is not in the defined range, is again set to zero. In the binarized image, the cell nuclei are white pixels. Connected regions of white pixels represent a nucleus and are labeled with a connected-component labeling algorithm (MATLAB function bwlabel). First, connected regions are determined using the Moore neighborhood (8 neighbors). Afterwards, distinct numbers are assigned to the regions, which label them in a unique way. For this, the union find algorithm is applied [14]. For determining the important parameter of cell migration depth, the shortest distance from a detected cell nucleus to the surface of the scaffold was calculated. The coordinates of the center of a labeled nucleus are utilized as the position of the cell. The coordinates $E_{x, i}$ and $E_{y, i}$ of the surface line of the scaffold are identified using a canny edge detector (MATLAB function edge) [15]. An example is given in Figure 8.

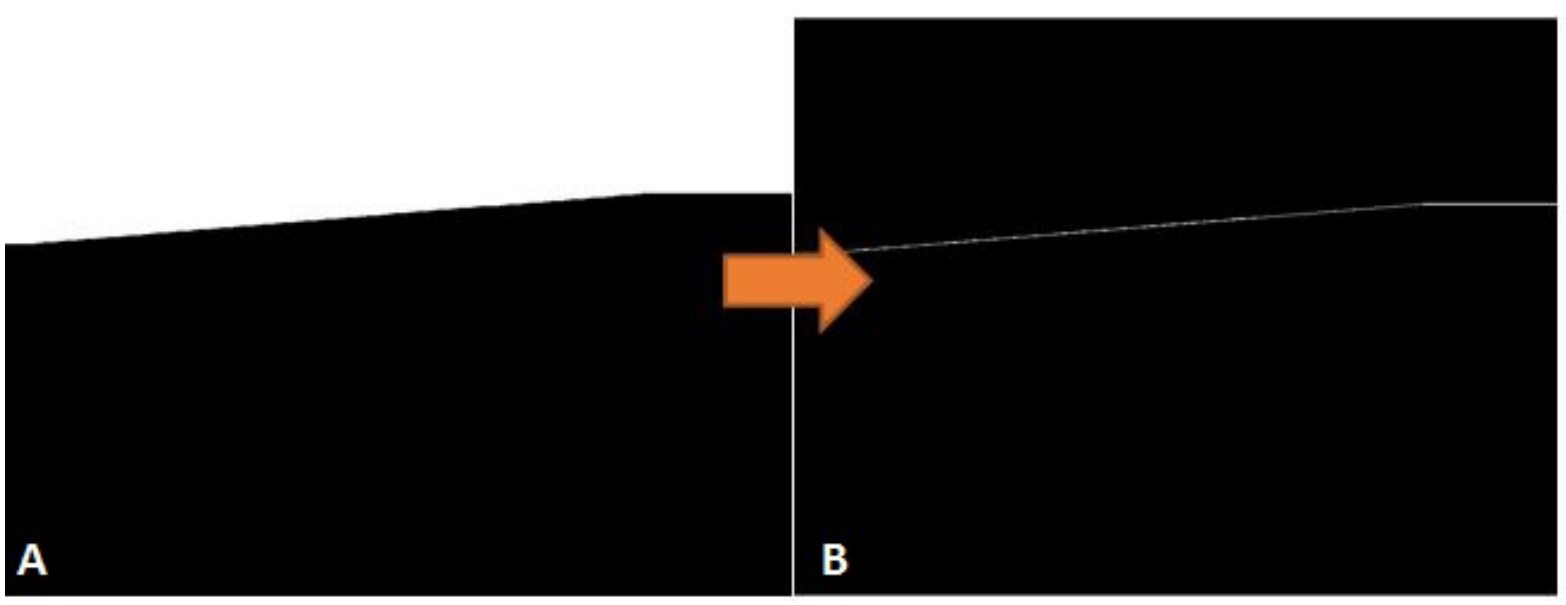

Figure 8: Binary image $(A)$, which represents the scaffold is in black. Using the canny operator, the surface of the scaffold is detected, which results in a new image $B$.

If $\mathrm{N}$ represents the number of white pixels of the edge line, $\mathrm{N}$ different distances can be calculated between one nucleus $\left(N_{x}, N_{y}\right)$ and the edge line pixels $\left(E_{x, i}, E_{y, i}\right)$ of the surface line:

$$
D[i]=\sqrt{\left(E_{x, i}-N_{x}\right)^{2}+\left(E_{y, i}-N_{y}\right)^{2}}, i=1, \ldots, N
$$

The shortest distance from $D[i]$ is selected as the distance from the nucleus to the surface. Figure 9 shows the result of the detected nuclei as well as the determined distances to the edge. 


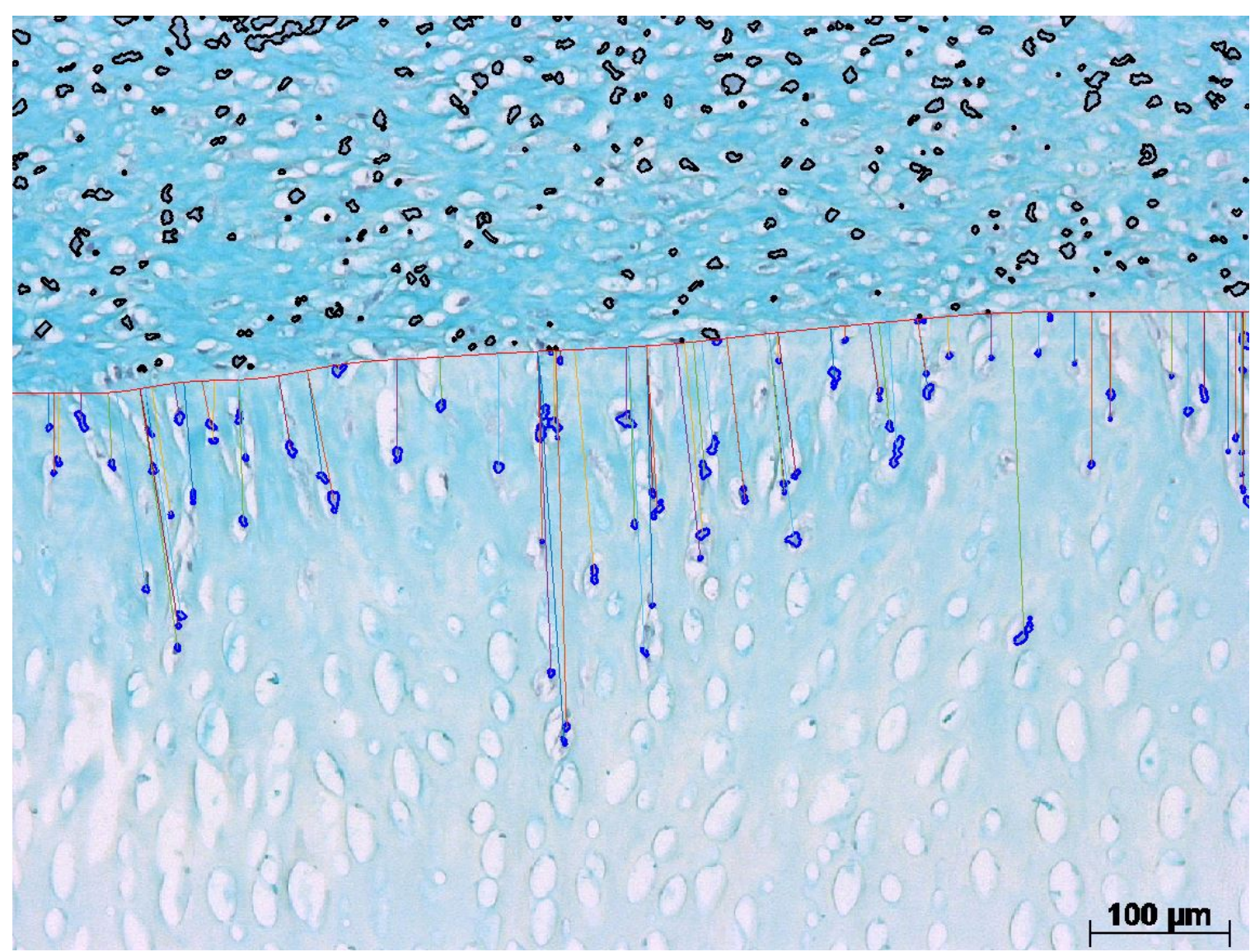

Figure 9: Image of an alcian blue stained sample with detected nuclei in the cell pellet (black framed) and inside the scaffold (dark blue framed). The red horizontal line represents the border between the scaffold and the cell pellet. The lines, which connect the red horizontal line with the nuclei, represent the shortest distances.

\subsection{Lacunae Analysis}

On the basis that the decellularized scaffold matrix mainly consists of collagen type II, the samples are brown colored after immunohistochemical staining of collagen type II. Because of the high contrast between the brown stained scaffold and the bright lacunae, this staining was employed to determine the orientation and density of the lacunae in the scaffold, which are supposed to influence the cell proliferation and migration. In Figure 10 the principle of lacunae labeling is presented with Figure $10 \mathrm{~A}$ as an example. In the binarized and corrected image in Figure $10 \mathrm{C}$ the lacunae can be distinguished. This is done using the major axis of a corresponding ellipse that has the same second moments as the region (MATLAB function regionprops). Likewise, the major and minor axis length and the number of pixels of each lacuna are calculated. 
Thomas Lepiarz, Ulla Wenzel, Michael Munz, Katja Hasch, Eva Goldberg-Bockhorn, Nicole Rotter, Martin Hessling; Computational Analysis of Histological Images of Tissue Engineered Cartilage for Evaluation of Scaffold Cell Migration. Journal of Biomedical Engineering and Medical Imaging, Volume 4, No 6, December (2017), pp 1-15

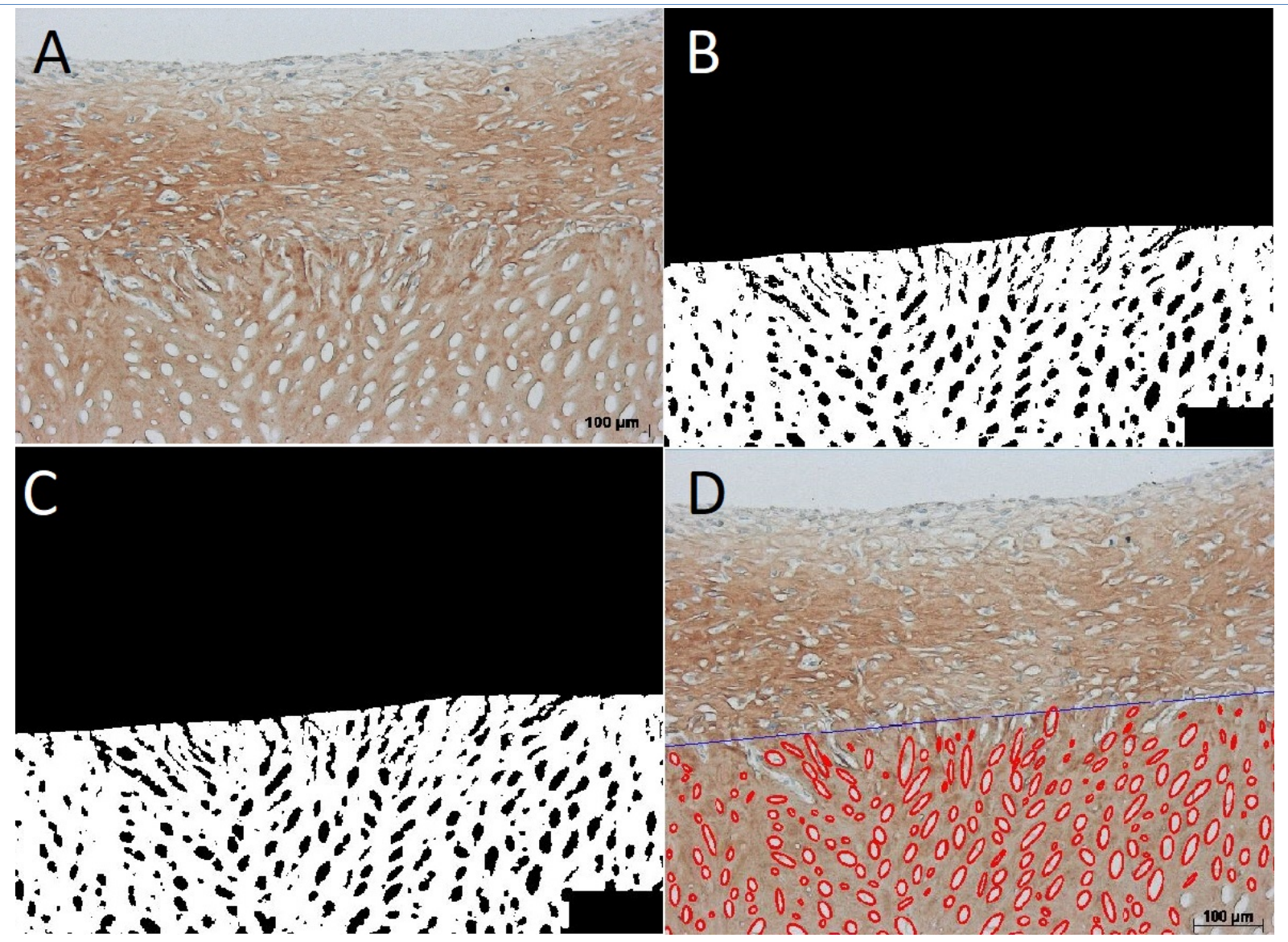

Figure 10: Principle of lacunae detection with original image (A) as an example. Detected lacunae (B) are corrected with erosion and dilatation (C) and labeled as red ellipsis (D).

Since the orientation of the detected lacunae is determined relative to the $x$-axes of the image, the orientation must be corrected relative to the surface of the scaffold. A straight line is fitted into the surface pixels (MATLAB function polyfit), resulting in the slope $k$ of the surface line. The orientation of the lacunae relative to this surface can then be corrected with the slope angle $\alpha$ :

$$
\alpha=\arctan (k) \cdot \frac{180}{\pi}
$$




\section{Results and Discussion}

The results of each segmentation algorithm were presented to an expert, who was asked to assess the detected ROIs and results of the software. According to the expert the results of the algorithm were comprehensible and correct. A more precise comparison between the software results and the evaluation of the expert is difficult, because the human expert does not generate quantitative values.

For additional validation of the quality of the results, the outcome has been compared to the results of different conventional laboratory methods. The total number of cells in and on the scaffold was determined with the QuantiFluor dsKits (Promega, Fitchburg; WI, USA) as described in [8]. The total content of Glycosaminoglycans (GAG) in and on the scaffold was measured by the Dimethylmethylene Blue (DMMB) assay [16].

In Figure 11 the relation between the detected cell nuclei with the segmentation algorithm and the determined cell number with QuantiFluor assay is presented. A dot represents the average of 6 samples.

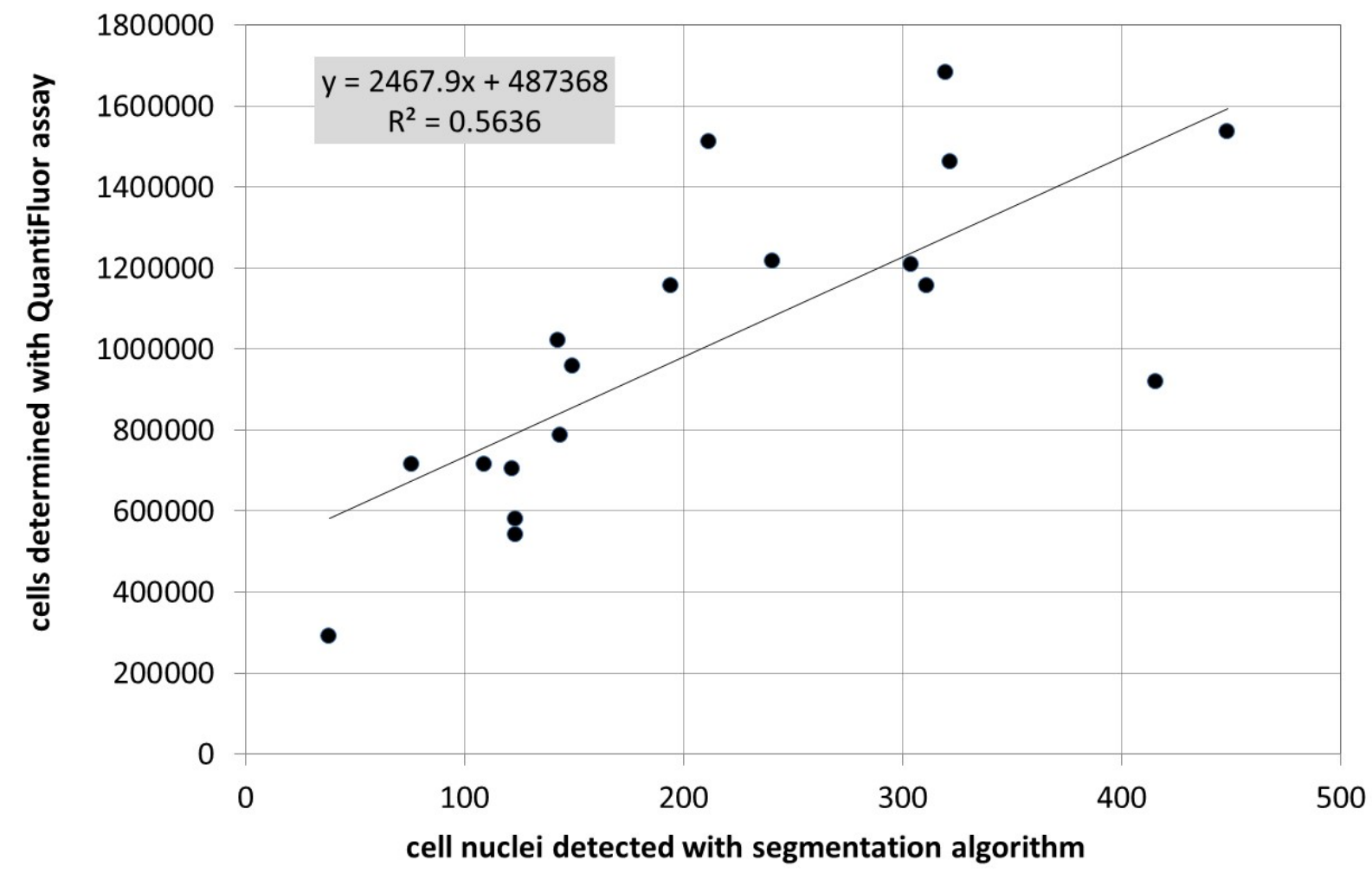

Figure 11: Comparison between the cell count detected by QuantiFluor assay and the number of cell nuclei identified by segmentation algorithm.

A correlation is clearly visible though the correlation coefficient seems not to be very high with $R^{2}=0.5636$. However, it should be considered that these biological samples show a high scatter even within the laboratory results. Additionally, it must be emphasized that the laboratory methods and the algorithms do not evaluate the same samples but only comparable samples, because each scaffold sample can only 
Thomas Lepiarz, Ulla Wenzel, Michael Munz, Katja Hasch, Eva Goldberg-Bockhorn, Nicole Rotter, Martin Hessling; Computational Analysis of Histological Images of Tissue Engineered Cartilage for Evaluation of Scaffold Cell Migration. Journal of Biomedical Engineering and Medical Imaging, Volume 4, No 6, December (2017), pp 1-15

be used either for staining or for laboratory analysis, but the same sample cannot be used for a direct comparison of both techniques. Regarding these boundary conditions, the correlation is quite reasonable.

Especially important is the fact, that this image analysis approach is the first method to distinguish between cells in the pellet on the scaffold surface and the much more important cells within the scaffold, which is not possible with the QuantiFluor assay or similar laboratory methods. The differentiation between the cell count in the pellet on the scaffold's surface and the migrated cells inside the scaffold can be important, if different TE methods are compared to improve the migration of the chondrocytes into a scaffold.

Another important value for cartilage quality or chondrocyte differentiation are the glycosaminoglycan (GAG) and aggrecan concentrations, as these polysaccharides and proteins are relevant components of cartilage produced by vital and well differentiated chondrocytes. The DMMB assay gives GAG content in $\mu \mathrm{g}$ per scaffold. The developed algorithm delivers a different value: a relative area in $\mu \mathrm{m}^{2}$ that is stained and detected as aggrecan. If both methods are valid they should exhibit a siginificant correlation. This is depicted in Figure 12.

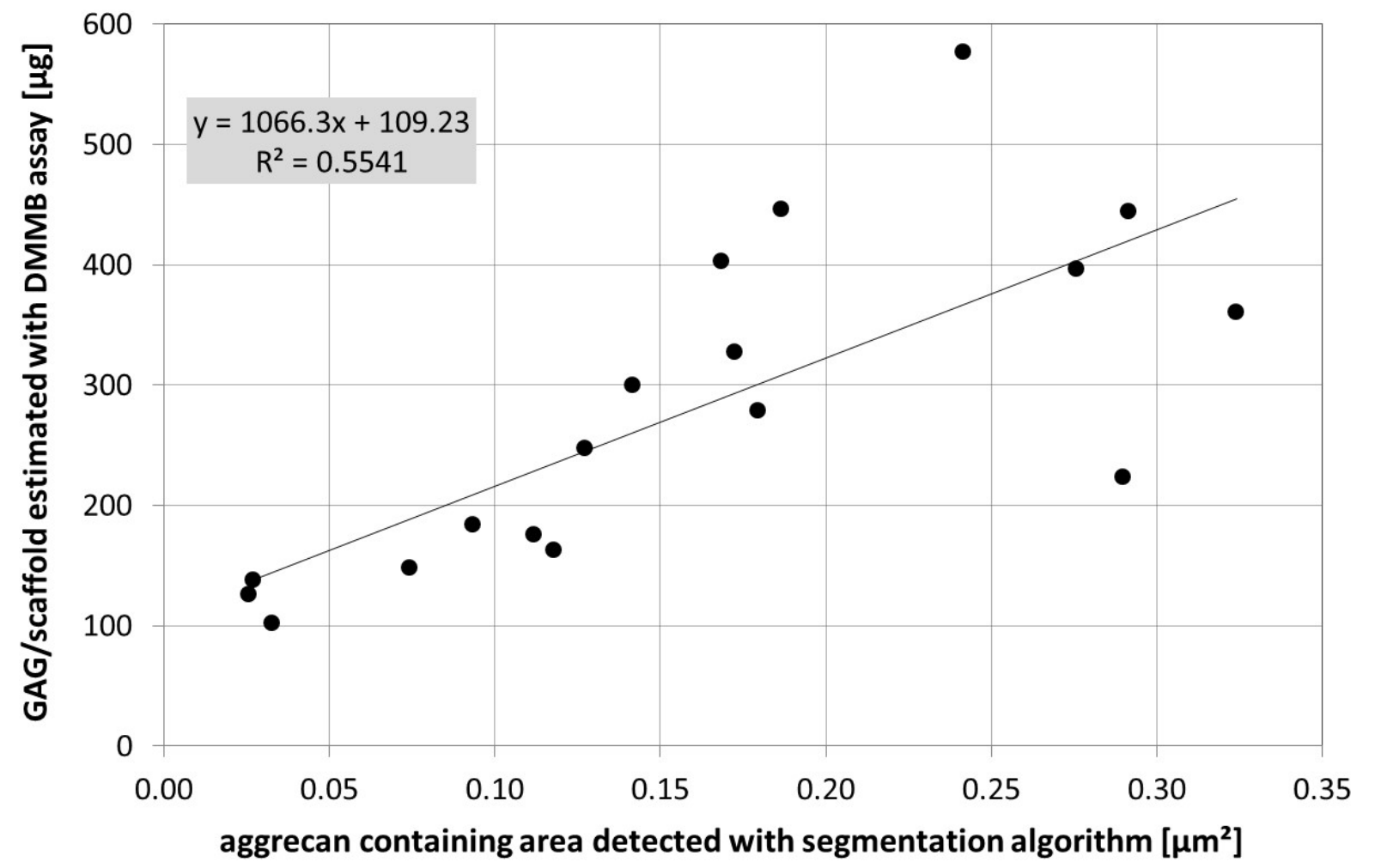

Figure 12: Content of GAG in $\mu \mathrm{g} / \mathrm{scaffold}$ determined by DMMB assay in comparison to the aggrecan containing area defined by segmentation algorithm.

It should be repeated that the biological samples are highly variable and both methods were applied to similar but not the same samples. This is an important reason for the seemingly low correlation coefficient of $R^{2}=0.5541$. But even with this restriction there is a pronounced correlation that confirms the algorithm results. 
In total 85 different images of three cultivation runs in the bioreactor and three cultivations under static conditions were analyzed with the segmentation algorithm, which is able to determine cell nuclei, aggrecan and collagen I on and in the scaffold separately. Additionally, the orientation, the size and the density of lacunae in collagen type II images is given as well as the migration depth of cell nuclei. For example: In Figure 9253 cell nuclei were detected in the cell pellet and 97 in the scaffold. The average migration depth of the cells is $86 \mu \mathrm{m}$ while the largest detected distance is $287 \mu \mathrm{m}$. Figure $10 \mathrm{D}$ shows 214 detected lacunae with an average orientation of $57,7^{\circ}$ relative to the scaffold surface. This newly obtained data will be analyzed and investigated in more detail in a future paper.

\section{Conclusion}

This research work presents a novel approach of image segmentation algorithm, which seems to deliver fast and meaningful results for the determination of cell numbers in and on top of the scaffold, migration depths, aggrecan and collagen type I concentrations as well as the number of lacunae and their orientation relative to the scaffold's surface. To our knowledge, it is the first and only semi-automatic technique that determines migration depths and lacuna properties. It also seems to be the first segmentation algorithm, which separates the detection of cell nuclei, aggrecan and collagen type I between scaffold and cell pellet. The algorithm will be employed to support the assessment of cultivation methods and conditions. Further investigation will be performed to find out if the density, the size and the orientation of the lacunae have an influence on cell migration and growth. More images will be analyzed to validate the results of the algorithm, which will be also refined to improve the reliability of the method.

\section{References}

[1] N. Rotter, M. Bücheler, A. Haisch et al., "Cartilage tissue engineering using resorbable scaffolds," Journal of Tissue Engineering and Regenerative Medicine, vol. 1, no. 6, pp. 411-416, 2007.

[2] A. Salerno, E. Di Maio, S. lannace et al., "Tailoring the pore structure of PCL scaffolds for tissue engineering prepared via gas foaming of multi-phase blends," Journal of Porous Materials, vol. 19, no. 2, pp. 181-188, 2012

[3] S. J. Hollister, "Porous scaffold design for tissue engineering," Nature Materials, vol. 4, no. 7, pp. 518-524, 2005.

[4] S. Schwarz, L. Koerber, A. F. Elsaesser et al., "Decellularized cartilage matrix as a novel biomatrix for cartilage tissue-engineering applications," Tissue Engineering. Part A, vol. 18, 21-22, pp. 21952209, 2012.

[5] M. N. Gurcan, L. E. Boucheron, A. Can et al., "Histopathological image analysis: A review," IEEE Reviews in Biomedical Engineering, vol. 2, pp. 147-171, 2009.

[6] J. M. Haggerty, X. N. Wang, A. Dickinson et al., "Segmentation of epidermal tissue with histopathological damage in images of haematoxylin and eosin stained human skin," BMC Medical Imaging, vol. 14, p. 7, 2014.

[7] T. J. Fuchs and J. M. Buhmann, "Computational pathology: Challenges and promises for tissue analysis," Computerized Medical Imaging and Graphics, vol. 35, no. 7, pp. 515-530, 2011.

[8] G. Matheron, Random sets and integral geometry, Wiley, New York, 1975. 
Thomas Lepiarz, Ulla Wenzel, Michael Munz, Katja Hasch, Eva Goldberg-Bockhorn, Nicole Rotter, Martin Hessling; Computational Analysis of Histological Images of Tissue Engineered Cartilage for Evaluation of Scaffold Cell Migration. Journal of Biomedical Engineering and Medical Imaging, Volume 4, No 6, December (2017), pp 1-15

[9] S. Princz, U. Wenzel, H. Tritschler et al., "Automated bioreactor system for cartilage tissue engineering of human primary nasal septal chondrocytes," Biomedizinische Technik. Biomedical Engineering, vol. 62, no. 5, pp. 481-486, 2017.

[10] S. Princz, U. Wenzel, S. Schwarz et al., "New bioreactor vessel for tissue engineering of human nasal septal chondrocytes," Current Directions in Biomedical Engineering, vol. 2, no. 1, p. 1415, 2016.

[11] A. F. Elsaesser, C. Bermueller, S. Schwarz et al., "In vitro cytotoxicity and in vivo effects of a decellularized xenogeneic collagen scaffold in nasal cartilage repair," Tissue Engineering. Part A, vol. 20, 11-12, pp. 1668-1678, 2014.

[12] A. F. Elsaesser, S. Schwarz, H. Joos et al., "Characterization of a migrative subpopulation of adult human nasoseptal chondrocytes with progenitor cell features and their potential for in vivo cartilage regeneration strategies," Cell \& Bioscience, vol. 6, p. 11, 2016.

[13] K. ElDahshan, M. Youssef, E. Masameer et al., "Comparison of Segmentation Framework on Digital Microscope Images for Acute Lymphoblastic Leukemia Diagnosis Using RGB and HSV Color Spaces," Journal of Biomedical Engineering and Medical Imaging, vol. 2, no. 2, 2015.

[14] R. Sedgewick, Fundamentals, data structures, sorting, searching, Addison-Wesley, Boston, Mass., 2009.

[15] J. Canny, "A computational approach to edge detection," IEEE Transactions on Pattern Analysis and Machine Intelligence, vol. 8, no. 6, pp. 679-698, 1986.

[16] I. Barbosa, S. Garcia, V. Barbier-Chassefière et al., "Improved and simple micro assay for sulfated glycosaminoglycans quantification in biological extracts and its use in skin and muscle tissue studies," Glycobiology, vol. 13, no. 9, pp. 647-653, 2003. 